\title{
. \\ Design of Redundant Degrees of Freedom in Task Realization of a Robot System with Positioning Errors of Objects*
}

\author{
Jia CHENG ${ }^{* *}$, Lounell B. GUETA**, Tamio ARAI***, Ryosuke CHIBA ${ }^{\dagger}$, \\ Tsuyoshi UEYAMA ${ }^{\dagger \dagger}$ and Jun OTA** \\ ${ }^{* *}$ Research into Artifacts, Center for Engineering, The University of Tokyo \\ 5-1-5 Kashiwanoha, Kashiwa-shi, Chiba 227-8568, JAPAN \\ E-mail: ota@ race.u-tokyo.ac.jp \\ *** Department of Precision Engineering, School of Engineering, The University of Tokyo \\ 7-3-1 Hongo, Bunkyo-ku, Tokyo 113-8656, JAPAN \\ $\dagger$ Faculty of System Design, Tokyo Metropolitan University \\ 6-6 Asahigaoka, Hino-shi, Tokyo 191-0065, JAPAN \\ $\dagger$ DENSO WAVE INCORPORATED \\ 1, Yoshiike, Kusaki, Agui-cho, Chita-gun, Aichi 470-2297, JAPAN
}

\begin{abstract}
This paper presents a method to minimize the task completion time of a manipulator system in a multiple-goal task by considering the existence of position errors of objects that may change the manipulator pose (position and orientation) and cause collisions. In this paper, we propose a method which cannot only reduce this change but also minimize the task completion time of the system. The proposed method resolves the redundancy of the system and the redundancy in the given task using Particle Swarm Optimization under a manipulability restriction. The proposed method is verified through simulations and is shown to be effective.
\end{abstract}

Key words : Manipulator Motion Planning, Multiple-goal Task

\section{Introduction}

The minimum completion time of a task is very important to improve production efficiency. Recently, manipulator systems used in multiple-goal tasks such as automatic inspection and automatic welding have garnered attention due to their operational accuracy and high flexibility. For example, a system composed of a manipulator and a positioning table is commonly used to achieve high efficiency ${ }^{(7),(8)}$. Ahmad proposed a motion-generation method for a system composed of a 2-degree-of-freedom (DOF) positioning table and a 7-DOF manipulator applied to automatic welding work ${ }^{(1)}$. In Ref. (6), a multiple-goal task is realized using a system composed of a 6-DOF manipulator and a 1-DOF positioning table, as shown in Fig.1, wherein the redundant DOFs of the system is utilized to minimize the task-completion time. The following factors, however, are essential at a production site.

(a) Degrees of freedom other than the redundant DOF of the system may exist depending on the given task. As shown in Fig.2, in an inspection, when pictures of an object are taken using a camera installed in the manipulator end effector, images can be captured at various orientations along the z-axis (i.e., the approach angle shown in Fig.2). Further, the camera can capture a clear image despite a difference in the position along the z-axis (i.e., the camerato-object distance shown in Fig.2). Since these positions and orientations do not need to be fixed, there is indeed extra DOFs in performing the task. In this paper, we call this as the redundancy in the task content. We conjecture that the task-completion time can be shortened by effectively resolving this redundancy.

${ }^{*}$ Received 2 Apr., 2010 (No. 10-0141) [DOI: 10.1299/jamdsm.4.1234]

Copyright (c) 2010 by JSME 


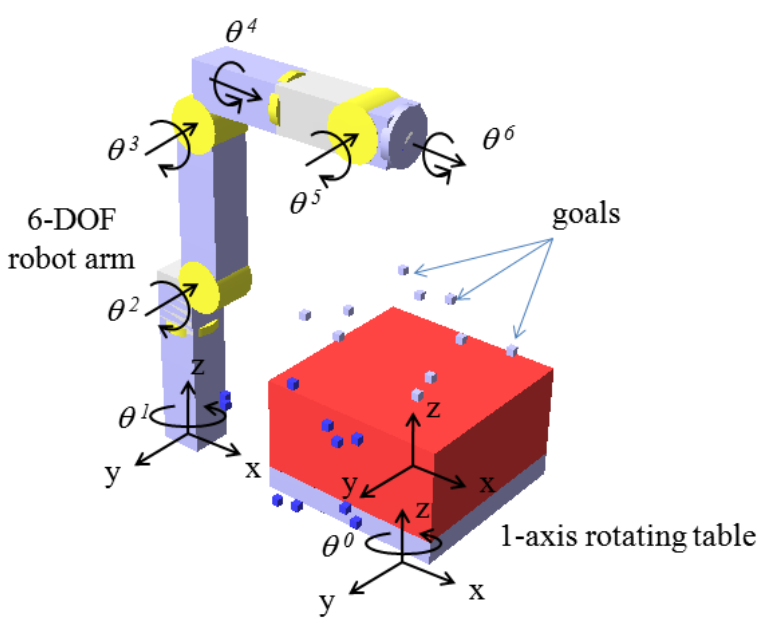

(a) Robot arm and table coordinate frames

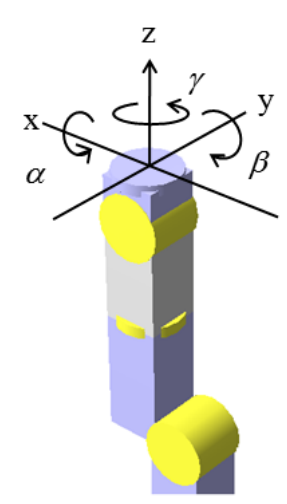

(b) Reference frame of the end-effector orientation

Fig. 1 A setup with a 6-DOF manipulator and a 1-axis positioning table. The end effector will move to several goals which are predefined parts of the object while the table positions the object.

(b) At a production site, an object is manually placed on a table that may lead to erroneous positioning. Since goals are defined relative to the object, their positions will also change relative to their original positions. Without the positioning error, the manipulator endeffector can reach these goals exactly at their original positions. With the positioning error, however, the manipulator end-effector position may fail to reach these positions. For instance, if the manipulator would reach a goal, a gap between the manipulator end-effector and goal may exists, making it impossible to execute task accurately. In this paper, such an error is referred to as the object-positioning error. In Ref.(2), a method is devised wherein a sensor detects the positioning error and feeds the information back to the manipulator for error correction. A small object-positioning error, however, may cause a remarkable increase in the task completion time. Moreover, when positioning errors are detected during an actual operation, motion generation of the manipulator system has to be carried out upon detection.

In the previous studies, the redundancy in the task content and the existence of objectpositioning errors have not yet been considered. Also, the manipulator system is kinematically redundant. In this study, a method is proposed to resolve these two types of redundancies using the Particle Swarm Optimization(PSO), thereby minimizing the task-completion time of the

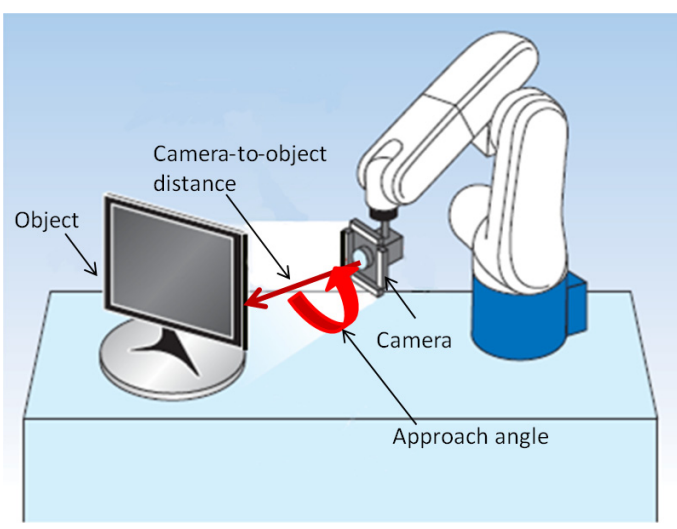

Fig. 2 A manipulator-based inspection system (Figure in Ref. (14) with modifications). 
system.

In dealing with object-positioning error, the robust optimization technique can be applied but may not be practical in terms of the calculation time requirement. In this study, a methodology that avoids the worst case, i.e., a deviation outside the manipulator joint limit, is adopted. This methodology is made feasible by imposing an inequality condition to avoid an increase in the task-completion time. Assuming the range of positioning errors can be known beforehand, the maximum posture change of the manipulator due to these errors can be derived. The worst case can then be avoided if the maximum posture change can be suppressed within a certain range. In this paper, a manipulability restriction is imposed to prevent a substantial posture change of the manipulator in reaching from one goal to the next goal.

Section 2 describes the previous studies. In Section 3, the problem is formulated. The proposed method is provided in Section 4. The experiment and results are discussed in Section 5 , and the conclusion is given in Section 6.

\section{Related works}

In this section, we introduce two groups of relevant works: (1) motion coordination of manipulator system and (2) robust optimization of the multivariable optimization problem.

\subsection{Motion coordination of manipulator system}

In Ref. (1), a 7-DOF manipulator with a 2-axis rotating table is utilized in a welding task wherein a continuous trajectory of the end- effector is given. The redundant DOFs of the system is used to minimize the task completion time. Similarly, a welding task is considered in Ref. (15) utilizing a system consisting of a 6-DOF manipulator and a 3-axis rotating table.

In Ref. (6), motion planning is combined with goal rearrangement to realize an inspection task. The arrangement of goals is formulated as a traveling salesman problem (TSP). To minimize the calculation time requirement, clustering of goals is proposed based on goal positions relative to the object to be inspected. For the motion planning, no modification of a straight-line path in the configuration space of the manipulator and table is conducted. The kinematic redundancy of the system is used to select the system configuration that has a collision-free path.

The above-mentioned works provide minimum-time motion-coordination methods of kinematically redundant systems, but the redundancy in the task content is not taken into consideration. Further, these works do not take into account posture changes of manipulator as a result of the positioning errors of objects.

\subsection{Robust optimization of the multivariable optimization problem}

Positioning error in the object placement may exists in a real work environment. This uncertainty must be considered in the motion generation of the manipulator system. If the task-completion time after the posture changes of the manipulator due to the error can be presumed, a min-max optimization ${ }^{(2)}$ can be used to minimize the presumed maximum taskcompletion time.

The method of presuming the task completion time includes response surface methodology(RSM) and linear approximation. In RSM, an error is generated randomly, and the task completion time is obtained as a response output ${ }^{(4)}$. The response surface can be constructed by taking the least square error of the output. In the presence of the errors, the task completion time of the manipulator system is thought to be on this response surface. If there is a linear relationship between the posture changes of the manipulator and the positioning errors, the maximum task-completion time of the system can be derived without constructing the response surface.

The RSM or linear approximation method can be used to estimate the task-completion time after the posture changes of the manipulator. This method, however, can require a large calculation cost due to the large number of design variables in this present study. 


\section{Problem statement}

Fig. 1 shows the manipulator system used in this study. Let $B_{o}, B_{t}$, and $B_{r}$ denote the object, table, and manipulator, respectively. Since $B_{t}$ and $B_{r}$ compose one redundant system, their configurations at a goal are denoted compactly as $q=\left\{\theta_{0} \cdots \theta_{6}\right\}$, where $\theta_{0}$ is the rotation angle of $B_{t}$ and $\left\{\theta_{1} \cdots \theta_{6}\right\}$ are the joint angles of $B_{r}$.

The goals are denoted as $p_{1}, \cdots, p_{N}$, where $N$ is the number of goals. A goal is a vector with position defined relative to $B_{o}$ and orientation as the required orientation of the end effector when $B_{r}$ reaches a goal. A goal is denoted as ${ }^{o} p_{i}=\left({ }^{o} \vec{r}_{i},{ }^{o} \alpha_{i},{ }^{o} \beta_{i},{ }^{o} \gamma_{i}\right), i \in\{1 \cdots N\}$ where ${ }^{o} \vec{r}_{i}=\left({ }^{o} x_{i},{ }^{o} y_{i},{ }^{o} z_{i}\right)$ is the position of the end effector, and ${ }^{o} \alpha_{i},{ }^{o} \beta_{i}$, and ${ }^{o} \gamma_{i}$ are the roll, pitch, and yaw, respectively. The start and end configurations of both $B_{r}$ and $B_{t}$ before and after all the goals are reached are the same, which is referred to as $q_{0}$.

In regards to the positioning of $B_{o}$, the errors can possibly occur along the x-axis $\Delta x$, along the $y$-axis $\Delta y$, and the rotation error about the z-axis $\Delta \gamma$. Because the positional relationship between the goals and the object does not change, these errors can be conversely expressed as errors at the goal position defined as $\overrightarrow{\delta p}$ where $\overrightarrow{\delta p}=(\Delta x, \Delta y, 0,0,0, \Delta \gamma)$ and is bounded by $-\delta p_{\max }(j)<\delta p(j)<\delta p_{\max }(j), j \in\{1, \cdots, 6\}$, where $j$ is the manipulator joint $j$ and $\delta \overrightarrow{p_{\text {max }}}$ denotes the maximum positioning error of the object.

\subsection{Input parameters}

The input parameters in this study are enumerated below.

- DH parameters of the manipulator and the joint limits of the manipulator

- Number of goals $N$ and the position of each goal $\vec{p}_{1}, \cdots, \vec{p}_{N}$

- Initial manipulator and table $\vec{q}_{0}$

- Positioning errors of $B_{o}, \delta p(j)$ and the maximum error $\delta p_{\max }(j), j \in\{1, \cdots, 6\}$.

Note that the positioning errors $\overrightarrow{\delta p}$ can be detected just before the task begins. Moreover, the $\delta p_{\max }(j), j \in\{1, \cdots, 6\}$ can be set beforehand.

\subsection{Problem description}

The task of the manipulator manipulator is to reach all goals based on the required goal position and orientation. The total point-to-point motion time in reaching all these goals must be minimized which can be calculated as:

$$
T=\sum_{i=0}^{N-1} c\left(p_{i}, p_{i+1}\right)+c\left(p_{N}, p_{0}\right)
$$

where $T$ is the task-completion time and $c(a, b)=\max _{j \in\{0, \cdots, 6\}}\left[t_{j}(a, b)\right]$ is the motion time from goal $a$ to goal $b$, and $t_{j}(a, b)=\mid\left(\theta_{a}^{j}-\theta_{b}^{j} \mid / V^{j, m a x}\right.$ where $\theta_{a}^{j}$ is the angle of joint $j$ at goal $a$, and $V^{j, m a x}$ is the maximum velocity of manipulator joint $j$.

As shown in Fig.1, the approach angle is the rotation about the z-axis of the end-effector, $\phi$, and the camera-to-object distance is the position along the $\mathrm{z}$-axis, $d z$. In addition, the rotation angle of $B_{t}$ is a redundant DOF of the system. These parameters are referred to as $\mathcal{X}_{i}=\left(\theta_{0 i}, \phi_{i}, d z_{i}\right), i \in\{1 \cdots N\}$. Since $X_{i}$ must be designed for every goal, the total number of design parameters is $3 N$. Note that the relationship between the object position and the goals is constant regardless of the positioning errors.

When positioning error exists, $\overrightarrow{\vec{p}}_{i}$ can be calculated as $\vec{p}_{i}+\overrightarrow{\delta p}$ where $\overrightarrow{\delta p}$ is assumed to be detected by a sensor. Since $T$ is a function of $\vec{p}$ as shown in (1), $T$ changes as $\vec{p}$ changes. Therefore, the problem in this study is formulated as an optimization problem with $\mathcal{X}$ as design variables to minimize $T$.

In some multiple-goal tasks like inspection, the goal order as well as the trajectory of the manipulator end-effector between two goals is not given in advance. Further, the redundant DOF in the task content and the redundant DOF of the system have to be resolved to derive the configuration of the manipulator system. Therefore, aside from designing $\mathcal{X}$, the goal order and the configuration of the manipulator $q$ and table at every goal is also designed. 


\section{Proposed method}

This section discusses the proposed method of calculating the manipulator system configuration with redundant DOFs( i.e., redundancy in the task content and kinematic redundancy) and under the existence of object-positioning errors.

\subsection{Overview of the proposed method}

As aforementioned, it is necessary to design the goal order as well as the configuration of the manipulator and table at each goal. The proposed method is shown in Fig.3. A goal order is determined first, and then the configuration at each goal is derived by resolving the redundant DOFs. We propose PSO as the method to design $\mathcal{X}_{i}, i \in\{1 \cdots N\}$. After deriving these solutions, the calculation time is checked whether the limit is reached or not. In this way, it is guaranteed that the solution of finding a goal order is derived with an associated solution from resolving the redundancy. Moreover, the positioning errors are taken into consideration to generate the collision-free path between goals. The process of determining the goal order and the manipulator system configuration is conducted iteratively until the design time limit is reached.

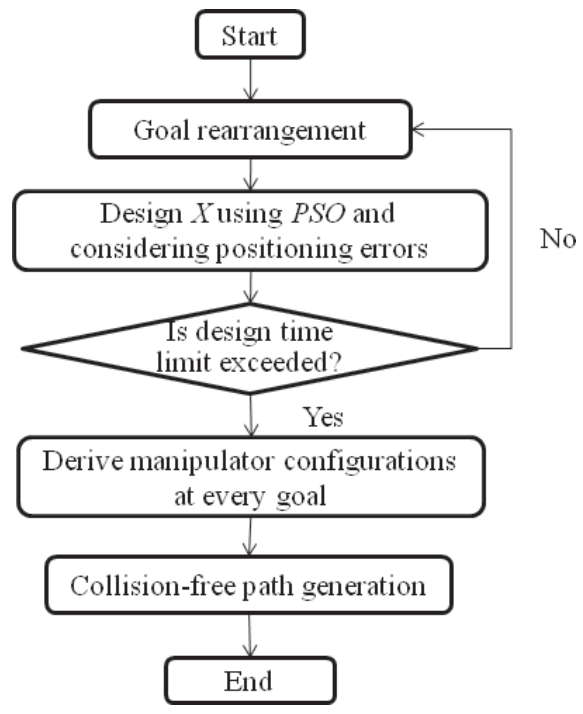

Fig. 3 Proposed method

The method shown in Fig.3 is proposed to derive practical solutions within a reasonable amount of calculation time since the problem at hand is too highly-dimensional. Solving the goal order is combinatorial in nature wherein there are $N$ ! possible orders for $N$ number of goals. In regard to redundancy, there are two types: kinematic redundancy as a result of having 7 DOFs and the redundancy in the task content. The former involves $7 N$ variables while the latter has $2 \mathrm{~N}$ variables. Moreover, a part of finding the solution is performing collision detection and deriving collision-free paths which are time-consuming processes.

Note that the goal rearrangement and designing $\mathcal{X}$ using PSO are solved separately. The goal rearrangement utilizes the method in Ref. (6). Being a discrete travelling salesman problem, the goal rearrangement cannot be solved simultaneously with designing $\mathcal{X}$ using PSO. Moreover, Ref. (6) cannot deal with the high dimensionality of the problem. Compared to Ref. (6), this current work has to deal with $2 N$ additional variables, which is due to designing the rotation about, and the translation along the approach axis of every goal.

Since a great deal of research has been done on collision-free motion planning ${ }^{(3),(9),(10)}$, motion planning is not the focus of this study. These previous research works, however, can be utilized in this research. 
In calculating the motion time from a goal to the next goal $c(i, j)$, the maximum velocity profile of each joint is utilized wherein every joint motion is calculated using the maximum joint speed. The motion time of the slowest joint is selected as the $c(i, j)$ value. It may seem that the joints are independently controlled; however, these joints are controlled in a coordinated manner. During collision detection, for instance, all joints of the manipulator achieve their individual final joint values simultaneously. The time to achieve the final joint values is determined by the calculated $c(i, j)$, which is dictated by the motion time of the slowest joint.

\subsection{Manipulability restriction}

When goal positions are changed due to the object-positioning error, the end-effector pose (i.e., position and orientation) changes accordingly. As shown in Fig.4, a small objectpositioning error can cause a manipulator joint to exceed its limit. The manipulator pose greatly changes leading to a substantial change in $T$. Moreover, when the manipulator pose exceeds the joint limits, it will not be able to execute the task completely.

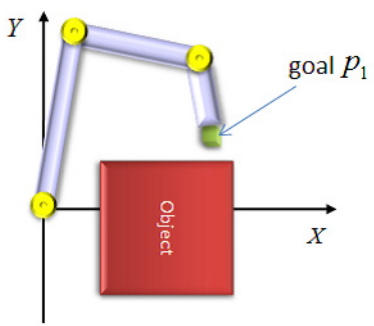

(a)

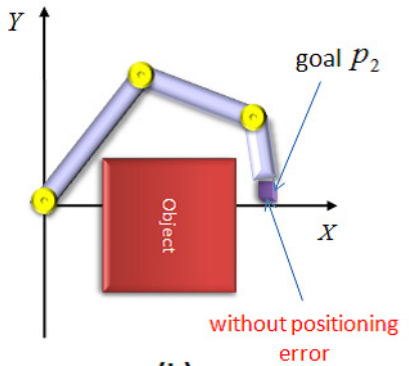

(b)

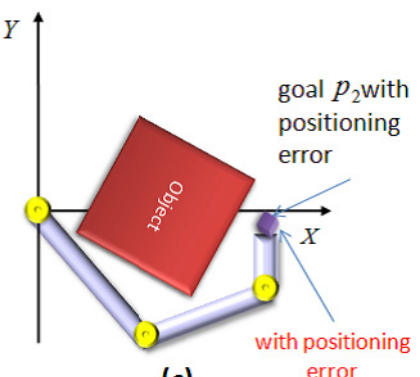

(c)

Fig. 4 Change of the manipulator pose brought by the object-positioning error

We conjectured that $T$ does not grow excessively if changes in the manipulator joint angles are kept within their limits. In addition, unsuccessful task execution can be prevented by restricting the manipulator joint rotation within limits. In this paper, such restriction is called the manipulability restriction.

Suppose $\Delta r$ denotes the change of the end-effector position due to object-positioning errors. The corresponding change in the manipulator configurations, $\Delta q$, can be derived as:

$$
\begin{aligned}
& \Delta r=J_{v}\left(q_{i}\right) \Delta q \\
& \Delta q=J_{v}\left(q_{i}\right)^{-1} \Delta r
\end{aligned}
$$

where $q_{i}, i \in\{1 \cdots N\}$ denotes the manipulator configuration in goal $i$ without the objectpositioning errors, and $J_{v}\left(q_{i}\right)$ is the jacobi matrix of the manipulator with configuration $q_{i}$. Because the change in the end-effector of the manipulator is the same as the change in the goal position, $\Delta r=\Delta p$.

The manipulability restriction can then be described as follows:

$$
q_{i}+\Delta q_{\max } \leq q_{\max , i}
$$


where $q_{\max , i}$ denotes the limit of the manipulator joint $j, \Delta q_{\max }$ is the change in the manipulator configuration when the object-positioning error $\delta p=\delta p_{\max }$. Note that (4) can be transformed to $\Delta q_{\max }=J_{v}\left(q_{i}\right)^{-1} \Delta r_{\max }$. Therefore, in deriving configuration $q_{i}$, the manipulability restriction described by (4) is imposed.

\subsection{Formulation and calculation of solution}

In deriving the manipulator system configuration, the manipulability restriction is imposed. That is, an optimization problem can be formulated with constraints, such as those shown in (5). The design parameter $\mathcal{X}_{i}=\left(\theta_{0 i}, \phi_{i}, d z_{i}\right), i \in\{1 \cdots N\}$ is designed under the manipulability restriction and a collision restriction so that the manipulator links do not collide with the object and table.

$$
\begin{array}{cl}
\text { minimize } & T=\sum_{i=0}^{N-1} c\left(p_{i}, p_{i+1}\right)+c\left(p_{N}, p_{0}\right) \\
\text { subject to } & h\left(\mathcal{X}_{i}\right)=0 \\
& q_{i} \leq q_{\max }-\Delta q_{i, \max }
\end{array}
$$

In (5), $h\left(\mathcal{X}_{i}\right)$ is a function that returns 0 or 1 such that $h\left(\mathcal{X}_{i}\right)=0$ means that no collision occurred. In this paper, we solve the problem shown in (5) by using PSO.

In order to minimize $T$, PSO is used to design $\mathcal{X}_{i}$ for the following reasons. It has been shown that PSO provides superior performance in deriving the global optimal solution to a multi-peak continuous function ${ }^{(12)}$. Moreover, PSO algorithm is simpler than GA algorithms in terms of tuning parameters. For details of the PSO algorithm, please refer to Ref. (5), (12).

In this study, the design parameter $\mathcal{X}_{i}=\left(\theta_{0 i}, \phi_{i}, d z_{i}\right), i \in\{1 \cdots N\}$ is the position vector of a particle in PSO. Fig. 5 shows an illustration of PSO. The subscript refers to a particle number while the superscripts $k-1, k$ and $k+1$ refer to the time evolution of particles. The variable $x$ is the position vector while $v$ is the velocity vector. Note that $x$ represents the design parameters in this study. The pbest is the best-known position of a particle while gbest is the best-position of the entire particles. For example, at time $k$, pbest $t_{1}^{k}$ is the best-known position of particle 1 while $g b e s t^{k}$ is the best-known position of all particles.

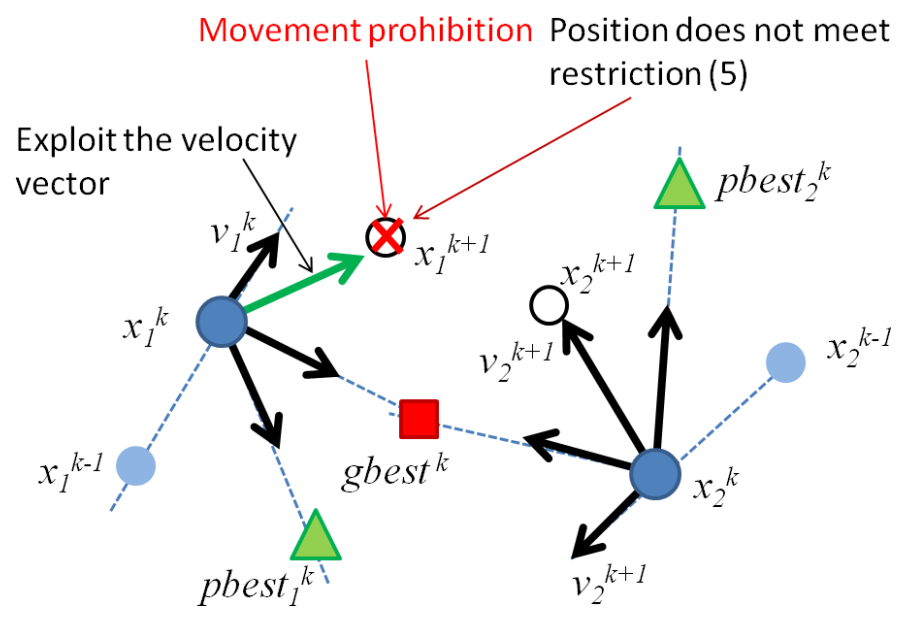

Fig. 5 Improvement of searching performance

Many PSO parameter-tuning methods have been proposed. Since the Linearly Decreasing $V_{\max }$ Method (LDVM) is shown to have the best search efficiency, it is used in this study. The manipulator configuration at each goal can be derived by solving the inverse kinematics using the derived value of $\mathcal{X}_{i}$. 


\subsection{Performance improvement of the search method}

It is necessary to design the manipulator configuration in each goal to satisfy the collision restriction and the manipulability restriction. As shown in Fig.5, these restrictions are satisfied by prohibiting the movement of the particle to the positions with collision and positions that do not satisfy the manipulability restriction.

The search space narrows when such a movement limitation is added. As a result, the optimum solution near a position that prohibits movement may not be found. The performance of PSO in terms of finding hig-quality solution is then decreased due to this movement prohibition. To prevent this problem, the velocity vector of the particle in the current position is updated, and the satisfaction of the restriction in (5) is ignored.

\section{Simulations, result and discussion}

In this section, the effectiveness of the proposed method is verified through simulations.

\subsection{Minimizing the task-completion time}

One of the purposes of this study is to resolve redundancy in the task content in order to reduce the task completion time of the system.

5.1.1. Simulation setting The proposed method, the optimization of the design parameter $\mathcal{X}_{i}=\left(\theta_{0 i}, \phi_{i}, d z_{i}\right)$ using PSO, is evaluated against the method in (Ref. (6)), which is referred to as the previous work. In the previous work, the rotation angle of the table is only designed using the nearest neighbor algorithm . Each method is applied to three objects with $N=30,40$, and 50. The values of some parameters are provided in Table $\mathbf{1}$.

Table 1 Design parameter setting

\begin{tabular}{c|c}
\hline Parameter & Setting \\
\hline$\theta_{0}[\mathrm{rad}]$ & $-\pi \sim \pi$ \\
$d z[\mathrm{~mm}]$ & $-10 \sim 10$ \\
$\phi[\mathrm{rad}]$ & $-\pi \sim \pi$ \\
\hline
\end{tabular}

5.1.2. Result and discussion

Fig. 6 shows the performance of the compared methods. For $N=30,40$, and 50, the task-completion time of the system was reduced by the proposed method.

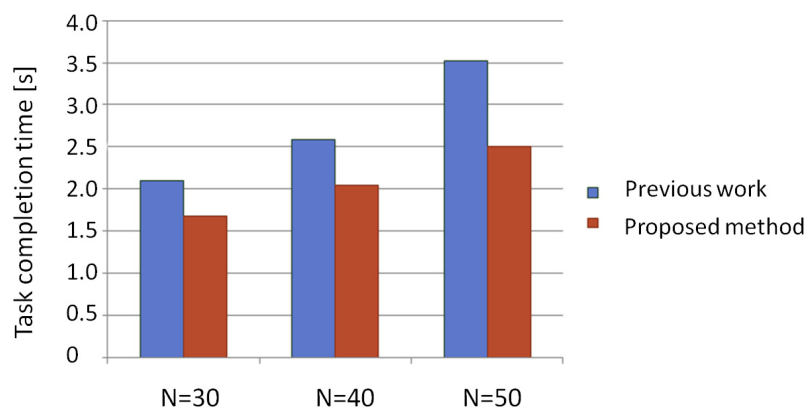

Fig. 6 A comparison of derived task-completion time of various search methods in $\mathrm{N}=30,40,50$

As an example, we examined the case of $N=30$ in detail. Fig.7 shows the motion time of the manipulator system $c\left(p_{i-1}, p_{i}\right)$, defined in Eq.(1). The motion slows greatly at goals No.10 No.11 and No.20 No.21 in the previous work. These motion times are shortened by the proposed method, leading to a decrease in the entire task completion time. As shown in (1), the motion time is proportional to the variation of the manipulator configurations, which was suppressed by the proposed method. 


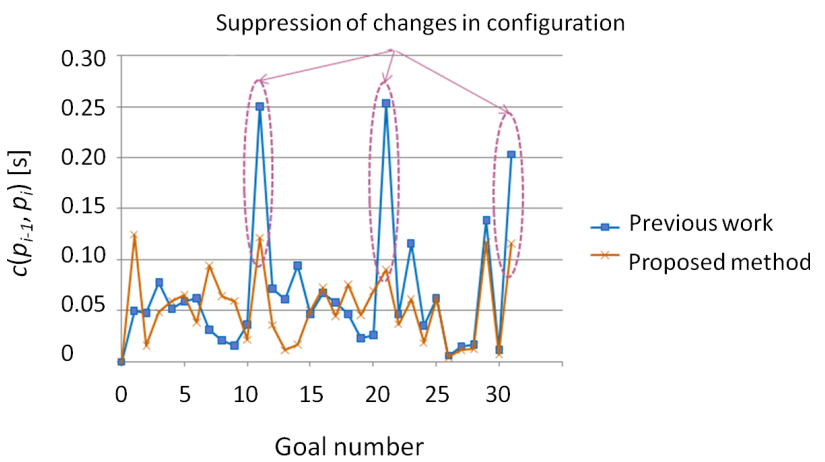

Fig. 7 A comparison of the derived motion of various search methods, $\mathrm{N}=30$

\subsection{Verifying the effectiveness of imposing the manipulability restriction}

A simulation is conducted to verify the effectiveness of imposing the manipulability restriction. In this simulation, we have the following conjectures.

i. When the manipulator system motion is generated using the proposed method, the worst case, i.e., the excessive increase in the task-completion time of the system, and interruption due to the singularity of the manipulator configuration and due to unreachable area, can be avoided.

ii. When the manipulator system motion is generated using the proposed method, the computational time must be less than that of the the min-max robustness optimization.

5.2.1. Simulation setting The following methods are compared to obtain the manipulator system configuration at each goal.

- Motion generation by optimizing the design parameter $\mathcal{X}_{i}=\left(\theta_{0 i}, \phi_{i}\right)$ only, (MG).

- Motion generation by optimizing the design parameter $\mathcal{X}_{i}=\left(\theta_{0 i}, \phi_{i}\right)$ using min-max robust optimization, (MGRO).

- Motion generation by optimizing the design parameter $\mathcal{X}_{i}=\left(\theta_{0 i}, \phi_{i}\right)$ with a manipulability restriction, (MGMR), the proposed method.

Recall that when maximum positioning errors exist, a maximum posture change of the manipulator occurs. As the positioning errors $\overrightarrow{\delta p}=(\Delta x, \Delta y, 0,0,0, \Delta \gamma)$ can be a positive maximum or a negative maximum, $2^{3}=8$ patterns of maximum positioning errors might exist. In robust optimization, we optimize the design parameter to minimize the task completion time for each pattern of maximum positioning errors. The solution is the one that gives the maximum task completion time.

The frequency at which the manipulator system stops functioning and the task completion time of the manipulator system derived by each method are compared. The positioning errors $\overrightarrow{\delta p}=(\Delta x, \Delta y, 0,0,0, \Delta \gamma)$ are randomly generated 100 times in the error range shown in Table 2. The collision-free path connecting each goal is generated using the method described in Ref. (9). Moreover, only two DOFs (i.e., the rotation angle of the table and the rotation about the approach angle) are considered as the design parameter. The limit value of each joint angle of the manipulator is shown in Table 3.

Table 2 Range of positioning errors

\begin{tabular}{c|c}
\hline Parameter & Setting \\
\hline$\Delta x[\mathrm{~mm}]$ & $-10 \sim 10$ \\
$\Delta y[\mathrm{~mm}]$ & $-10 \sim 10$ \\
$\Delta y[\mathrm{deg}]$ & $-10 \sim 10$ \\
\hline
\end{tabular}

5.2.2. Results and discussion The results of the simulation are shown in Table 4. In the motion generated by MG, the manipulator system did not complete the task once for $N=30$, 28 times for $N=40$ and 6 times for $N=50$. On the other hand, in the motion generated by 
Table 3 Joint limit value of the manipulator

\begin{tabular}{c|c|c|c}
\hline Joint & Range & Joint & Range \\
\hline$\theta_{1}$ & $-170^{\circ} \sim 170^{\circ}$ & $\theta_{4}$ & $-190^{\circ} \sim 190^{\circ}$ \\
$\theta_{2}$ & $-100^{\circ} \sim 135^{\circ}$ & $\theta_{5}$ & $-120^{\circ} \sim 120^{\circ}$ \\
$\theta_{3}$ & $-119^{\circ} \sim 169^{\circ}$ & $\theta_{6}$ & $-360^{\circ} \sim 360^{\circ}$ \\
\hline
\end{tabular}

MGMR, the task is executed completely. The cause of unsuccessful completion of tasks is that no solution in the manipulator inverse kinematics is possible at the 27th goal. In Table 4, we verified the effectiveness of the proposed method (MGMR) wherein the case when the manipulator system failed to complete the task due to positioning errors was avoided.

Table 4 Frequency of unsuccessful task completion

\begin{tabular}{|l|c|c|c|}
\hline Compared methods & $N=30$ & $N=40$ & $N=50$ \\
\hline MG & 1 & 28 & 6 \\
\hline MGRO & 0 & 0 & 0 \\
\hline MGMR & 0 & 0 & 0 \\
\hline
\end{tabular}

An example of a task with $N=40$ is examined in detail. For the positioning errors $\Delta x=-4.551[\mathrm{~mm}], \Delta y=-1.125[\mathrm{~mm}]$, and $\Delta \gamma=0.137[\mathrm{deg}]$, the motions generated by MG and MGMR are shown in Fig.8 and Fig.9, respectively. In these figures, the limit value of the 5th joint angle of the manipulator is plotted as shown by the red line. The inset shows the manipulator configuration at the 26th goal.

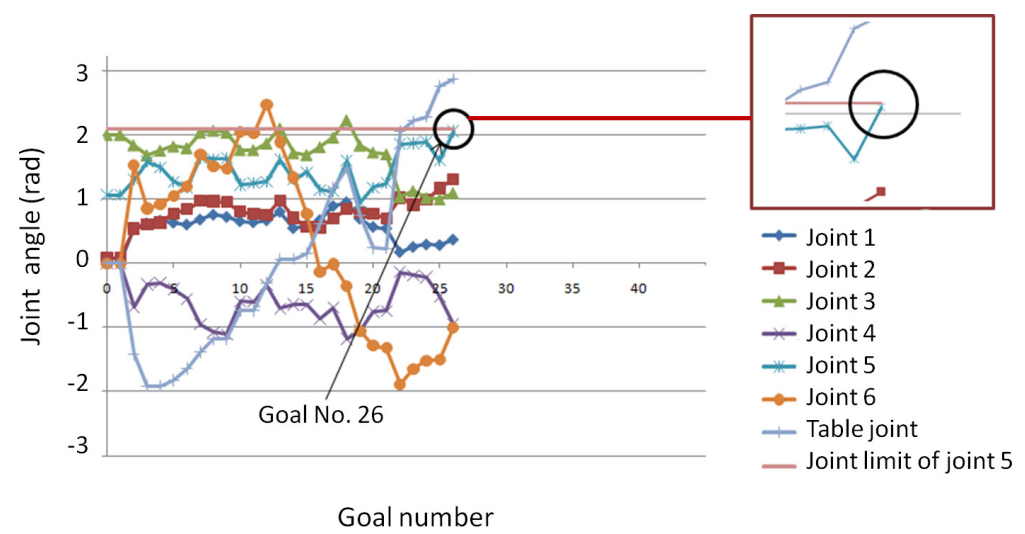

Fig. 8 Configuration of manipulator system at each goal (MG, N=40)

Fig.8 shows that the manipulator system ceases to complete the task in the motion generated by MG. It only shows configurations from the 1st goal to the 26th goal. On the other hand, the motion generated by MGMR for $N=40$ successfully completed the task as shown in Fig.9.

As shown in Fig.8, the 5th joint angle of the manipulator approaches the limit at the 26th goal with MG. At this configuration, it is difficult to rotate the 5th manipulator joint clockwise in order to move toward the 27th goal. On the other hand, as shown in Fig.9, with motion generation using MGMR, the 5th joint angle of the manipulator at the 26th goal is suppressed from reaching the joint angle limit.

Fig. 10 shows the snapshots of the manipulator system configuration at the 26th goal derived by MG and MGMR. The red lines indicate the angle value of joint 5 from when its value is zero to its positive joint angle limit, which is $120^{\circ}$ as shown in Table 3 . The black line shows the actual value of joint 5 . The suppression to reach the joint limit is depicted by a narrower angle of the 5th joint in MGMR compared to that of MG.

In this example, both the MGMR and MGRO successfully completed the task. In terms 


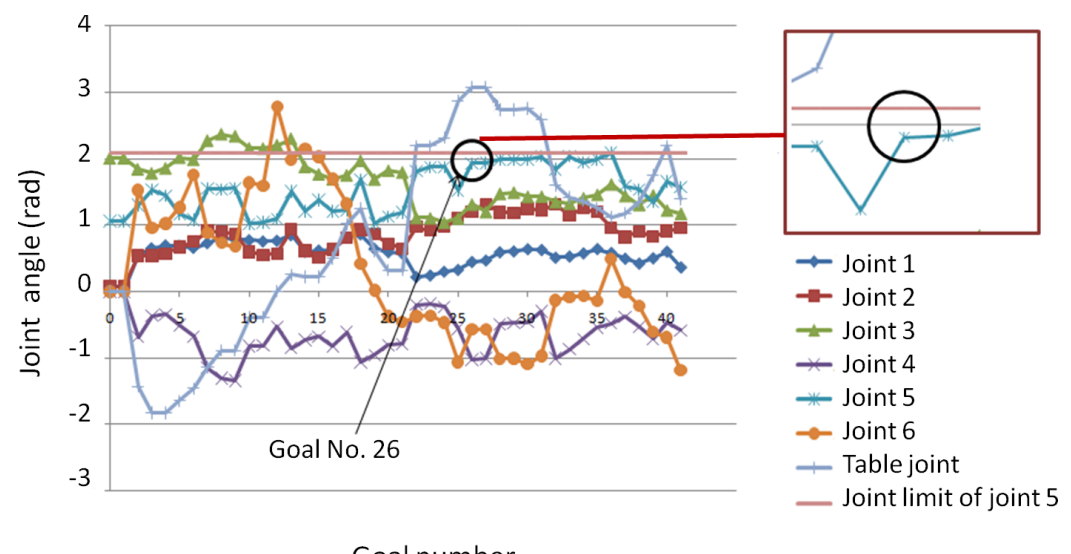

Goal number

Fig. 9 Configuration of the manipulator system at each goal (MGMR, N=40)

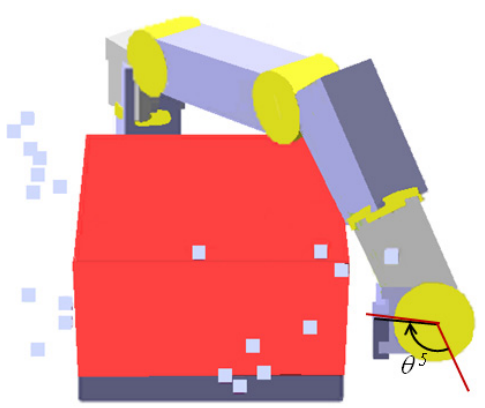

(a) $\mathrm{MG}$

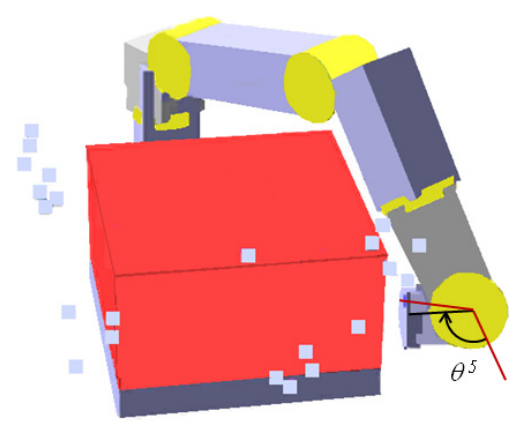

(b) MGMR

Fig. 10 Manipulator system configurations at the 26th goal

of the task completion time of the system, MGMR achieved 2.32[s], and MGRO was 3.23[s] showing that the former can reduce the task completion time more effectively than the latter.

In terms of the calculation time, the average time of calculating $\mathcal{X}=\left(\theta_{0}, \phi\right)$ at one goal is shown in Table 5. The result shows that the average calculation time of using MGMR is 2.45[s], which is about 3.4 faster than that of using MGRO.

Table 5 Average calculation time

\begin{tabular}{|c|c|c|c|}
\hline Compared methods & MG & MGRO & MGMR \\
\hline Calculation time (s) & 2.50 & 8.26 & 2.45 \\
\hline
\end{tabular}

\section{Conclusion}

In this paper, we propose a motion-generation method for a manipulator system consisting of a 6-DOF manipulator and 1-DOF positioning table. The positioning errors of the object are considered. Moreover, the redundancy in the task content as well as the kinematic redundancy are taken into account to achieve minimal task completion time. The problems are solved through the following approaches.

- In the problem formulation, a manipulability restriction of the manipulator is imposed based on the possible range of object positioning errors.

- The redundancy in the task content and the kinematic redundancy are resolved using PSO.

The change in the manipulator configuration due to the positioning errors was suppressed by the above-mentioned approach. 


\section{References}

( 1 ) Ahmad, S. and Luo, S., Coordinated motion control of multiple robotics devices for welding and redundancy coordination through constrained optimization in Cartesian space, IEEE Trans. on Robotics and Automation, Vol.5, No.4(1989), pp.409-417.

( 2 ) Averbakh, I., Minmax regret solutions for minmax optimization problems with uncertainty, Operations Research Letters, Vol. 27(2000), pp.57-65.

( 3 ) Bohlin, R. and Kavraki, L.E., Path planning using lazy PRM, Proceedings of IEEE International Conference on Robotics and Automation, (2000), pp. 521-528.

( 4 ) Dellino, G., Kleijnen, J.C.P., and Meloni, C., Robust optimization in simulation: Taguchi and response surface methodology, CentER Discussion Paper Series, No.200869(2008).

( 5 ) Fourie, P.C. and Groenwold, A.A., The particle swarm optimization algorithm in size and shape optimization, Structural and Multidisciplinary Optimization, Vol.23, No.4(2002), pp.259-267.

( 6 ) Gueta, L.B., Chiba, R., Ota, J., Ueyama, T., and Arai, T., Coordinated motion control of a robot arm and a positioning table with arrangement of multiple goals. Proceedings of the 2008 IEEE International Conference on Robotics and Automation, (2008), pp. 2252-2258.

( 7 ) Jouaneh, M.K. Wang, Z., and Dornfeld, D.A., Trajectory planning for coordinated motion of a robot and a positioning table: Part 1-path specification, IEEE Trans. on Robotics and Automation, Vol.6, No.6 (1990), pp.735-745.

( 8 ) Jouaneh, M.K., Dornfeld, D.A., and Tomizuka, M., Trajectory planning for coordinated motion of a robot and a positioning table: Part 2-optimal trajectory specification, IEEE Trans. on Robotics and Automation, Vol.6, No.6(1990), pp.735-745.

( 9 ) Kondo, K., Motion planning with six degrees of freedom by multistrategic bidirectional heuristic free-space enumeration, IEEE Transactions on Robotics and Automation, Vol.7, No.3(1991), pp.267-277.

(10) Kuffner, J.J. Jr. and LaValle, S.M., RRT-Connect: An efficient approach to single-query path planning, Proceedings of IEEE International Conference on Robotics and Automation, Vol. 2(2000), pp.995-1001.

(11) Raibert, M.H. and Craig, J.J., Hybrid position/force control of manipulator, ASME Journal of Dynamic Systems, Measurement, and Control, Vol. 103(1981), pp.126-133.

(12) Schutte, J.F. and Groenwold, A.A., A study of global optimization using particle swarms, Journal of Global Optimization, Vol. 31, No. 1(2005), pp.93-108.

(13) Song, G., Thomas, S., and Amato, N.M., A general framework for PRM motion planning, IEEE International Conference on Robotics and Automation, (2003), pp. 44454450 .

(14) Website of DENSO WAVE INCORPORATED. (online), available from http://www.denso-wave.com/ja/robot/product/latest/vir/, (accessed 2010-4-2).

(15) Wu, L., Cui, K., and Chen, S., Redundancy coordination of multiple robotic devices for welding through genetic algorithm, Robotica, Vol. 18(1999), pp.669-676. 